\title{
High Speed and Spectrally Efficient Optical Communication System - Review of the Recent Advances and Emerging Trends
}

\author{
Nitin Muchhal \\ Associate Professor \\ Sagar Institute of Science \& Technology SISTec, Bhopal \\ Aditi Bhatt \\ Associate Professor \\ Sagar Institute of Science \& Technology, Bhopal
}

\begin{abstract}
The increasing growth in the data traffic demands posed by recent advances in cloud computing, social networking and smart devices is rapidly saturating the capacity of deployed optical fiber infrastructure. According to recent forecasts $\sim 30 \%$ increase in internet traffic per year is expected which is quickly driving the optical networks out of capacity[1]. In order to avoid this capacity crunch, the overall capacity of the deployed optical fiber network must be increased. There has been continuous work to increase the capacity \& data rate beyond Terabit/second using various multiplexing technology. This paper presents the features and recent advances in technologies (wired/wireless)e.g., OFDM, WDM, SDM, Li-Fi being used in optical communication to enhance the data rate, spectral efficiency, bandwidth etc. with review on recent progress in each.
\end{abstract}

Keywords Optical OFDM, CO-OFDM, WDM, Li-Fi, Long Haul

\section{INTRODUCTION}

Communication of information has been a prime factor in the development of human civilization. From wired systems to wireless systems, there have been tremendous developments in the method of communication. Today Internet has become very major mode of communication and today around $40 \%$ of the world population has an internet connection which was less than 1\% In 1995[2]. As recent history has shown, the amount of data traffic these networks will carry will increase dramatically and continuously. This need of large bandwidth and high speed can only be coped up by Optical fiber communication (wired \& wireless). n principle, optical fibers offer a tremendous amount of potential transmission bandwidth or capacity, currently used for a wide range of applications, such as long-distance data transmission (e.g. internet traffic), fiber-to-the-home (FTTH) and cable television (CATV). Maximizing transmission capacity requires new optimized fiber designs and communication techniques, which have been developed over the last decades in multiple steps, beginning with increased bit rates in telecommunications applications. 


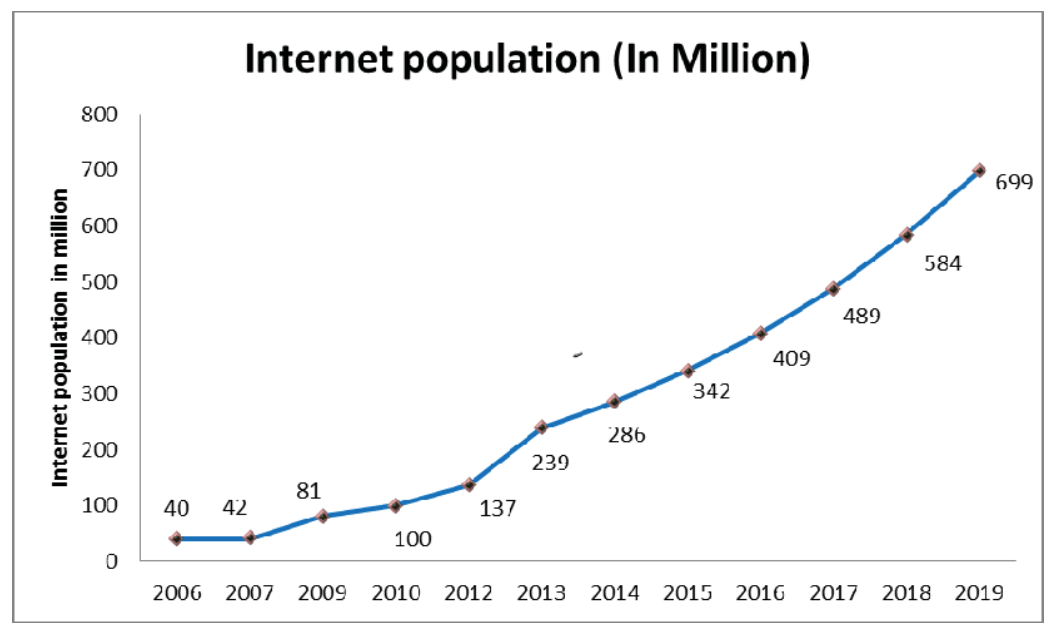

Fig.1 Increase in Internet Users

Although there are several ways of communication and they address to a very broad range of radio spectrum, but these technologies are still restricted due to the attenuation faced by the waves traveling through them. This problem was reduced by the development of guided/wireless optical communication [3]. The amount of attenuation faced by the light wave traveling from an optical fiber is much less compared to that of other communication medias. This facilitates the usage of low power devices, like LEDs and Lasers to be utilized as inputs for very long range (typically up-to tens of thousands of Kms) communication at a very high speed. Because of this reason, the optical communication revolutionized the existing communication technologies. It also led to the development of high speed modulation techniques to achieve desired data rates over these channels.

\section{VARIOUS MultipleXing TechniQues}

Fiber optical communication world will view capacity crisis thus innovative schemes have to be developed for capacity augment. For that, number of multiplexing techniques has been explored from time to time with their respective pros and cons. Here is brief review of various multiplexing techniques [4 ] being used for Optical communication.

\subsection{ORTHOGONAL FREQUENCY DIVISION MULTIPLEXING (OFDM)}

Among various modulation techniques, Orthogonal Frequency Division Multiplexing (OFDM) has emerged as a very popular optical communication technique [2]. OFDM is a derivative of Frequency Division Multiplexing (FDM). Over the last decade, OFDM has emerged as an important physical-layer signal transmission scheme in wireless communications [5]. OFDM based signal modulation has been utilized very widely in mobile communications including, digital audio and video broadcasting. This is due the fact attributed to its high speed, considerably low attenuation and its resistance to frequency selective fading, in comparison to other communication technologies. Even with such advanced characteristics, the current world yearns for more speed and data rate with least possible data loss. This continuously emerging need has lead to rapid developments in the area of optical communication and modulation. Orthogonal-FDM (OFDM) achieves this by sending multiple symbols over a set of subcarriers. To ensure orthogonality over a bit-period, each symbol is modulated using a unique combination of inphase (in phase with the carrier) and Quadrature (90 degree out of phase with the carrier) components. These components are allotted in such a way that they ensure maximum spectral efficiency over a symbol-period and are less affected by fading.

\section{DESIGN AND IMPLEMENTATION OF OFDM SYSTEM}

The generation of OFDM [6] signal started from serial to parallel converter. The serial data need to convert into parallel format, since QAM (Quadrature Amplitude Modulation) module requires parallel input . Such parallel data 
is mapped to appropriate symbol. The parallel symbols are transformed from frequency domain into time domain, using IFFT module. The data is converted into serial format for transmission.

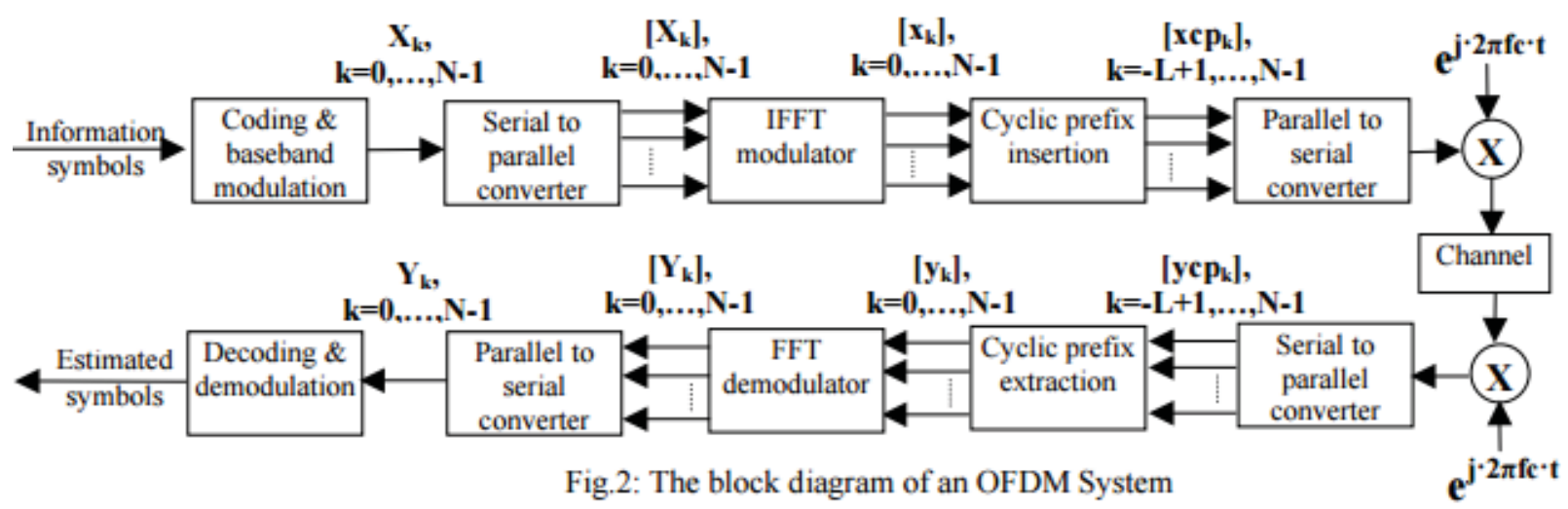

\subsubsection{Optical OFDM}

In general, electrical OFDM signals are complex valued data signals. To convert a complex valued data signal into the optical domain [7], the signal can be electrically up-converted using an electrical intermediate frequency (IF) carrier to modulate the signal upon applying a complex electrical I/Q mixer. The resulting up-converted electrical OFDM data signal can be electro-optic (e/o) converted using a state-of-the-art amplitude modulator. Alternatively, a complex electro- optic I/Q modulator can be driven directly by the complex electrical OFDM signal and thus converted into the optical domain. In optical receivers, typically, simple photodiodes are applied which operate according to the square law detection scheme. Since It is impossible to transfer the optical OFDM signal directly into the electrical domain. Instead of Direct detection of the pure optical OFDM signal, an optical carrier must be delivered either by the transmitter (using direct detection OFDM) or by a local oscillator in the receiver (using coherent optical OFDM) via a heterodyne or intradyne approach.

By comparing [8] CO-OFDM with DD-OFDM, we will find that the CO-OFDM provides the best robustness against chromatic dispersion $\mathrm{CD}$, and polarization mode dispersion PMD . This is because of the linear effect in coherent detection technique which improves the receiver sensitivity. On the contrary, the dispersion tolerance in DD-OFDM is limited because of the nonlinear direct detection. In addition, CO-OFDM is mostly used in long-haul applications due to the expensive and complex equipment used in the E/O and O/E conversion. In contrast, DDOFDM is cost effective solution for cost sensitive applications such as Local Area Networks LANs and Metropolitan Area Networks MANs

2.2 Wavelength Division Multiplexing (WDM) The wavelength -division multiplexing WDM [9]is a technology which multiplexes a number of optical carrier signals into a single optical fiber by using different wavelengths of laser light. This technique enables bidirectional communications over one stand of fiber, as well as multiplication of capacity, where each wavelength carries a separate channel. WDM divides the optical spectrum to smaller channels, which are used to transmit and receive data simultaneously. Figure illustrates the optical WDM networks, where wavelength substitutes frequency and each transmitter transmits separated wavelength $\lambda \mathrm{i}$, where $\mathrm{i}=1,2,3, \ldots . . \mathrm{N}$. 


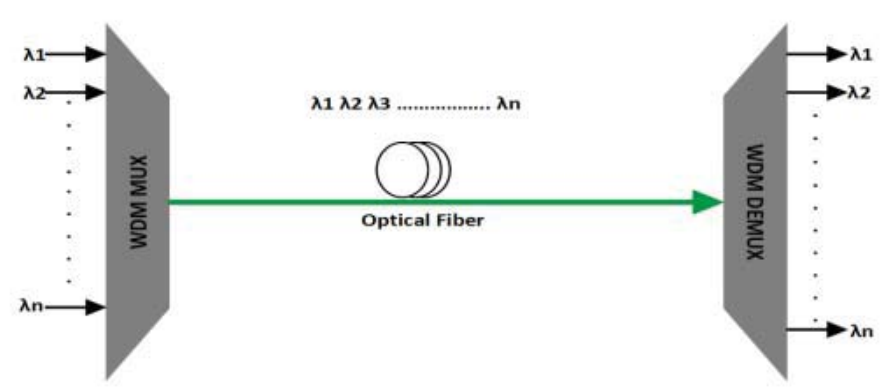

Fig. 3 wavelength division multiplexing

WDM systems include two types, Dense Wavelength Division Multiplexing (DWDM) and Coarse Wavelength Division Multiplexing (CWDM). The DWDM is the solution for huge demand in data in communications networks. DWDM connects the users' devices directly to the router which provides greater bandwidth By using DWDM, the transmission capacity and distance will increase by minimizing wavelength spacing. Another type of WDM is CWDM which is cost effective. CWDM is used for short transmission distances of less than $50 \mathrm{~km}$ because it is more cost efficient than the DWDM system.

\subsection{Space Division Multiplexing (SDM)}

The term SDM[10] now a day's refer to multiplexing techniques that establish multiple spatially distinguishable data pathways through the same fiber, although in earlier days the same terminology was previously applied to describe the case of multiple parallel fiber systems: the benchmark that needs to be beaten on a cost-per-bit perspective if any of the SDM approaches currently under investigation are ever to be commercially deployed. Space-division multiplexing (SDM) uses multiplicity of space channels to increase capacity for optical communication. It is applicable for optical communication in both free space and guided waves. The major new components for SDM are space-multiplexer to couple light from different cores or different modes into SDM fibers, SDM fibers, SDM optical amplifiers to amplify SDM signals, spaced de-multiplexer \& optical connectors.

\subsection{Polarization-Diversity Multiplexing (PDM)}

Polarization-division multiplexing (PDM) is a physical layer method for multiplexing signals carried on electromagnetic waves using thepolarization of the electromagnetic waves to distinguish between the different orthogonal signals. Polarization-division multiplexing is typically used together with phase modulation or optical QAM, allowing transmission speeds of $100 \mathrm{Gbit} / \mathrm{s}$ or more over a single wavelength.

\subsection{Mode Division Multiplexing (MDM)}

Amongst many of the multiplexing schemes mode-division multiplexing MDM [11] is one of the swiftly emerging scheme. It is getting more concentration due to maturity of digital signal processing, in a different way with growing capacity need over signals per fiber core. When multiple independent modes are used as an independent channel, the scheme is also called mode-division multiplexing (MDM).There has been much progress in MDM transmission experiments since 2011, employing well designed FMFs or coupled MCFs either with or without multiple-input multiple-output (MIMO) processing for long haul and high capacity. Lot of signals can be transmitted through propagation modes, on any particular core and particular wavelength. Researchers has also investigated about MDM-WDM[11] transmission with LP01 and two degenerate LP11 modes [12] for high speed communication.

\section{Literature ReView: ReCEnt DeVElopment in Optical Communication}

OFDM was introduced to optical domain in 2005[13] \& since then there has been rapid growth in transmission rate of signals. In year 2009, Takayuki et al [14 ] described novel electro-optic subcarrier multiplexing scheme to generate an over $100 \mathrm{~Gb} / \mathrm{s}$ high speed optical OFDM signal with a small number of subcarriers and successfully demonstrated transmission over 80-km of single-mode fiber without dispersion compensation. As reported in [15 ] 1 $\mathrm{Tb} / \mathrm{s}$ per channel is demonstrated using Coherent optical frequency-division multiplexing transmission. In year 2010 Shief et al [16] proposed a novel approach called multiband DFT-spread OFDM (MB-DFT-S-OFDM), by which the DFT-SOFDM is applied to each sub-band of the multiband CO-OFDM to reduce the PAPR within each sub-band \& 
achieved 107-Gb/s transmissions over long haul (1000-km) standard-single-mode-fiber (SSMF). In year 2011, Yu et al[17] proposed and experimentally demonstrated a novel scheme to generate coherent 21 optical subcarriers with fixed frequency of $25 \mathrm{GHz}$. Using these optical subcarriers, $1.96 \mathrm{~Tb} / \mathrm{s}(21 \times 100 \mathrm{~Gb} / \mathrm{s})$ polarization-multiplexing optical orthogonal frequency multiplexed (PM-OFDM) signal was generated. In similar year, Hillerkuss et al [18 ] demonstrated an optical fast Fourier transform scheme that provides the necessary computing power to encode lower-bit rates tributaries into 10.8 and 26.0 Tbit s -1 line-rate orthogonal frequency-division multiplexing (OFDM) data streams and to decode them from fiber-transmitted OFDM data streams. As reported in [19], Zhu et al described a new multicore fiber (MCF) having seven single-mode cores arranged in a hexagonal array, exhibiting low crosstalk among the cores and low loss across the C and L bands. We experimentally demonstrate a record transmission capacity of $112 \mathrm{~Tb} / \mathrm{s}$ over a $76.8-\mathrm{km}$ MCF using space-division multiplexing and dense wavelengthdivision multiplexing (DWDM). In same year [20] reported the first experimental demonstration of space-divisionmultiplexed DWDM transmission of PDM-QPSK channels over a multicore fiber \& transmitted a total capacity of $56-\mathrm{Tb} / \mathrm{s}(7 \times 80 \times 107-\mathrm{Gb} / \mathrm{s})$ over a $76.8-\mathrm{km}$ seven-core-fiber with a record spectral-efficiency of $14-\mathrm{b} / \mathrm{s} / \mathrm{Hz}$.. In [21] Qian et al demonstrated transmission of $101.7-\mathrm{Tb} / \mathrm{s}(370 \times 294-\mathrm{Gb} / \mathrm{s})$ over $3 \times 55 \mathrm{~km}$ SSMF using PDM-128QAMOFDM modulation and pilot-based phase noise mitigation and achieved spectral efficiency of $11 \mathrm{bits} / \mathrm{s} / \mathrm{Hz}$.

In year 2012, As reported in [22], the successful transmission of $64 \mathrm{~Tb} / \mathrm{s}$ capacity over $320 \mathrm{~km}$ reach utilizing $8-\mathrm{THz}$ of spectrum in the $\mathrm{C}+\mathrm{L}$-bands at a net spectral efficiency of $8 \mathrm{bit} / \mathrm{s} / \mathrm{Hz}$. It was accomplished by the use of raised-cosine pulse-shaped PDM-36QAM modulation, and ultra-large-area fiber where each 25-GHz channel were subdivided into four sub-bands, with each sub-band carrying a 73.5-Gb/s OFDM signal modulated with polarization-division-multiplexing (PDM) 128-ary Quadrature amplitude modulation (QAM) at each modulated subcarrier. In similar year, Xiang et al[23] discussed the generation and transmission of $450 \mathrm{~Gb} / \mathrm{s}$ wavelengthdivision multiplexed (WDM) channels over the standard $50 \mathrm{GHz}$ ITU-T grid optical network at a net spectral efficiency of $8.4 \mathrm{~b} / \mathrm{s} / \mathrm{Hz}$ by the use of Nyquist-shaped, polarization-division-multiplexed (PDM) 32-quadrature amplitude modulation (QAM) and both pre- and post-transmission digital equalization. Qian et al [24] experimentally demonstrated $101.7-\mathrm{Tb} / \mathrm{s}$ transmissions over $355 \mathrm{~km}$ spans of standard single-mode fiber (SSMF) at a net spectral efficiency of $11 \mathrm{~b} / \mathrm{s} / \mathrm{Hz}$ using 370 dense wavelength-division multiplexed channels spanning the optical C- and L-bands spaced at $25 \mathrm{GHz}$. In the same year, Sleiffer et al [25] demonstrated transmission of a 73.7 $\mathrm{Tb} / \mathrm{s}(96 \times 3 \times 256-\mathrm{Gb} / \mathrm{s})$ DP-16QAM mode-division-multiplexed signal over $119 \mathrm{~km}$ of few-mode fiber transmission line incorporating an inline multi mode EDFA and a phase plate based mode (de-)multiplexer.

In year 2013, Xiang et al (26) proposed the enabling modulation, coding, and line system technologies, as well as the existing challenges. It was shown that, $400 \mathrm{~Gb} / \mathrm{s}$ per channel signal can be transmitted on the standard 50 GHz. ITU-T grid at $8.4 \mathrm{~b} / \mathrm{ds} / \mathrm{Hz}$ net spectral efficiency (SE) over meaningful transmission reach for regional and metropolitan applications. Li et al [ 27] demonstrated $63-\mathrm{Tb} / \mathrm{s}(368 \times 183.3-\mathrm{Gb} / \mathrm{s})$ signal over $160-\mathrm{km}$ standard single mode fiber (SSMF) transmission in the C- and L-bands with 25-GHz channel spacing by using PDM-OFDM16QAM modulation, all-Raman amplification, coherent detection,. Also Foursa et al [28] transmitted 441x100Gb/s signals in $9 \mathrm{THz}$ of optical bandwidth over $9,100 \mathrm{~km}$ and a bit rate-distance product $>400 \mathrm{~Pb} / \mathrm{s} * \mathrm{~km}$. Fahad et al (29) in his work analyzed the integration of direct detection optical orthogonal frequency division multiplexing (DDOOFDM) with wavelength division multiplexing (WDM) to reach high data rates of $1.050 \mathrm{~Tb} / \mathrm{s}$ over $3600 \mathrm{~km}$ single mode fiber (SMF). In the report [30], Jun et al reported record capacity data transmission at $305 \mathrm{~Tb} / \mathrm{s}$ over $10.1 \mathrm{~km}$, using space division multiplexing (SDM) with 19 channels. They fabricated a trench-assisted homogeneous 19-core fiber \& also fabricated a 19-channel SDM multiplexer/demultiplexer using free-space optics with low insertion losses and low additional crosstalk. As reported in [31], Spectrally-efficient 17.6-Tb/s DWDM optical transmission system was demonstrated over $678 \mathrm{~km}$ with pre-filtering analysis.

In year 2014, Cai et al (32) proposed and designed a model to transmit $49.3 \mathrm{~Tb} / \mathrm{s}$ over 9,100 km using 282 16QAM coded modulation channels with spectral efficiency matched to the transmission system's spectral performance. In same year Guifang et al [33] reported work on SDM for fiber-optic communication using few-mode fibers or multimode fibers, in particular on the critical challenge of mode crosstalk. In year 2015, Sinan et al[34] analyzed a Coherent optical orthogonal frequency division multiplexing (CO-OFDM) system with dense wavelength division multiplexing (WDM) to reach high data rates of $1.60 \mathrm{~Tb} / \mathrm{s}$ over $4500 \mathrm{~km}$ single mode fiber (SMF). 1.60Tb/s signal was generated by multiplexing 16 OFDM signals with $100 \mathrm{~Gb} / \mathrm{s}$ for each signal. In same year, Rahman et al [35] discussed the trade-offs at transmitter and receiver to achieve long-haul reach for $1 \mathrm{~Tb} / \mathrm{s}$ superchannel. In year 2016, Xiao et al[36 ] successfully demonstrated four-channel wavelength-division multiplexing (WDM) $256.51 \mathrm{~Gb} / \mathrm{s}$ 16-ary quadrature amplitude modulation (16QAM)-OFDM signal transmission system for short-reach optical 
amplifier free inter-connection with real-time reception based on FPGA. Millar et al[ 37] described the design of a trained and pilot-aided digital coherent receiver, capable of detecting a $1 \mathrm{~Tb} / \mathrm{s}$ super channel with a single optical front end. Zhang et al demonstrated transmission capacities of 34.9Tb/s over $6375 \mathrm{~km}$ based on Gaussian-like DP64APSK and $33.3 \mathrm{~Tb} / \mathrm{s}$ over $6800 \mathrm{~km}$ using DP-32QAM are over hybrid spans with quasi-single-mode fiber. In 2016, Mizuno et al[ 38] described recent progress in space division multiplexed (SDM) transmission, and our proposal and demonstration of dense space-division multiplexing (DSDM), which offers the possibility of ultra-high capacity SDM transmission systems with high spatial density and spatial channel count of over 30 per fiber. Finally as reported in [39], Zhang et al demonstrated transmission capacities of $34.9 \mathrm{~Tb} / \mathrm{s}$ over $6375 \mathrm{~km}$ based on Gaussianlike DP-64APSK and $33.3 \mathrm{~Tb} / \mathrm{s}$ over $6800 \mathrm{~km}$ using DP-32QAM over hybrid spans with quasi-single-mode fiber.

\section{Li-Fi TeChNology: Wireless Data Transmission through Visible Light}

Light Fidelity (Li-Fi)[40] is a bidirectional, high-speed and fully networked wireless communication technology similar to Wi-Fi. The term was coined by Harald Haas[41] and is a form of visible light communication and a subset of optical wireless communications (OWC) and could be a complement to RF communication (Wi-Fi or cellular networks), or even a replacement in contexts of data broadcasting Li-Fi, basically known as "Light Fidelity" or "Optical Wi-Fi," is the technology of transmitting data using light waves using a LED light bulb that varying the light intensity faster than the human eye can follow. It is based on the visible light communication (VLC), which is a data communication medium for transmitting data using visible light in the range between $375 \mathrm{~nm}$ and $780 \mathrm{~nm}[42]$.

According to Haas, LEDs will evolve to more than just light sources and in the next 25 years, bulbs will have the processing power of modern cell phones. The Li-Fi Consortium [43] emphasizes that it is possible to get speeds greater than $10 \mathrm{GHz}$ using Li-Fi technology. In the beginning, over 500 megabytes per second (data rate) was achieved by researchers at the Heinrich Hertz Institute in Berlin in Germany using a stander white-light LED [44]. At the IEEE Photonics Conference[45] in October, Li-Fi consortium showed off the progress of combining both emitters and photodiodes to detect light using available red, green and blue LED's. By doing that the system could both send and receive data at aggregate rates of $110 \mathrm{Mbps}$. They developed their system to transmit data at the speed of $1.1 \mathrm{Gbps}$ over 10 meters. In 2013, Researchers at the Fraunhofer Henrich Hertz Institute (HHI) in Germany have successfully transmitted data at $3 \mathrm{Gbps}$ using conventional LED bulbs in a laboratory setting [46]

Sisoft (Mexican company) and researchers from Autonomous Technological Institute of Mexico (ITAM)[47] claimed that they have used LED lamps for transmitting data in one direction, with data rate 10Gbps, while simultaneously lighting the room. Recently, $224 \mathrm{Gbps}$ bi-directional speed over 3 meters was achieved by University of Oxford researchers using IR technology[48]. This speed will allow 18 movies, $1.5 \mathrm{~GB}$ each, to be downloaded in a single second. Also researchers at Leeds University achieved $20 \mathrm{Gbps}$, indoor VLC using laser diodes (LDs) [49]. Now recent research is going on in area of Solar Li Fi [50] where Light is used to transmit high speed data and the solar panel is LiFi-enabled to be a receiver - as well as energy harvesting, the solar panel provides energy for LiFi Technology and is a broadband receiver.

\section{CONCLUSION}

This paper covers detailed study on various multiplexing techniques for high speed wired and wireless optical communication. In recent years, the data rate over OFDM channel has increased tremendously from $100 \mathrm{~Gb} / \mathrm{s}$ to 100 $\mathrm{Tb} / \mathrm{s}$. The study in this paper suggests the contribution of various novel sub-band carrier modulations schemes in achieving this high data rate. Further, based on the study and analysis of various OFDM techniques, we noticed that the CO OFDM has come up as an efficient alternative in reducing the effects of frequency selective fading and dispersion. This adds to the advantage of having a faster response channel and good bandwidth efficiency. Also, we have noticed that the usage of high efficiency and precision equipments for the generation and detection of OFDM signals has helped a lot in achieving such high data rates. Also recent work on wireless optical communication ( $\mathrm{Li}$ Fi) has been reviewed which offers tremendous scope for future innovation and research. This technique will help to create the cleaner, safer, greener and a resplendent future by utilizing natural solar energy.

\section{REFERENCES}

[1] Cisco Visual Networking Index: Forecast and Methodology -2019

[2] Internet Growth Statistics - the Global Village Online

[3] Gerd Keiser, "Optical Fiber Communications”, Mc Graw-. Hill International edition, 4th Edition, 2000

[4] https://en.wikipedia.org/wiki/Multiplexing

[5] Wireless Communications, Andreas F. Molisch 2nd Edition 2010

[6] OFDM and Multi-Channel Communication Systems-National Instruments/white paper/3740 
[7] Fred Buchali, Roman Dischler, and Xiang Liu," Optical OFDM: A Promising High-Speed Optical Transport Technology" Bell Labs Technical Journal 14(1), 125-146 (2009)

[8] Nabih Mansor Abu Aloff, “Coherent OFDM for Optical Communication systems”, Master's Thesis 2014.

[9] https://nextgenerationoptical.com/

[10] D. J. Richardson1 , J. M. Fini," Space Division Multiplexing in Optical Fibres"Nature Photonics Review 2012 Berdague, S. \& Facq, P. Mode division multiplexing in optical fibers. Applied Optics, 21, (1982)

[11] A. Tarighat, R.C.J. Hsu, A. Shah, A.H. Sayed, B. Jalali, Fundamentals and challenges of optical multiple-input multiple-output multimode fiber links,IEEE Commun. Mag. (2007) 57-63.

[12] Qi Yang, Abdullah Al Amin, and William Shieh,” Impact of Nonlinearities on Fiber Optic Communications, Optical and Fiber Communications Reports chapter2" Springer, LLC 2011

[13] Takayuki Kobayashi, Akihide Sano, Eiichi Yamada, "Over $100 \mathrm{~Gb} / \mathrm{s}$ Electro-Optically Multiplexed OFDM for High-Capacity Optical Transport Network", JOURNAL OF LIGHTWAVE TECHNOLOGY, VOL.27, NO. 16, AUGUST 15, 2009

[14] Yan Tang; William Shieh "Coherent Optical OFDM Transmission Up to $1 \mathrm{~Tb} / \mathrm{s}$ per Channel", Journal of Lightwave Technology (Volume:27, Issue: 16 ), pp $3511-3517,2009$

[15] William Shieh and Yan Tang," Ultrahigh-Speed Signal Transmission Over Nonlinear and Dispersive Fiber Optic Channel: The Multicarrier Advantage", IEEE Photonics Journal, pp 276-285, Vol. 2, No. 3, June 2010

[16] J. Yu,; Z. Dong; N. Chi, "1.96 Tb/s (21 x 100 Gb/s) OFDM Optical Signal Generation and Transmission Over 3200-km Fiber", IEEE Photonics Technology Letters, Volume:23, Issue: 15 Page(s): 1061 - 1063, 2011

[17] D. Hillerkuss,R. Schmogrow,T. Schellinger,"26 Tbit s-1 line-rate super-channel transmission utilizing all-optical fast Fourier transform processing", Nature Photonics 5,pp364-371, 2011

[18] B. Zhu, T.F. Taunay, M. Fishteyn, X. Liu ,"112-Tb/s space-division multiplexed DWDM transmission with 14-b/s/Hz aggregate spectral efficiency over a 76.8-km seven-core fiber", Optics Express vol 19, Issue 17, pp. 16665-16671, 2011

[19] B Zhu, T Taunay, M Fishteyn, X Liu, "Space-, wavelength-, polarization-division multiplexed transmission of 56-Tb/s over a 76.8-km seven-core fiber", IEEE Optical Fiber Communication Conference, Los Angeles 2011

[20] D Qian, MF Huang, E Ip, YK Huang," 101.7-Tb/s (370× 294-Gb/s) PDM-128QAM-OFDM transmission over 3× 55-km SSMF using pilot-based phase noise mitigation", Optical Fiber Communication Conference and Exposition, Los Angeles, CA 2011

[21] ZTE COMMUNICATIONS- 100G and Beyond: Trends in Ultrahigh-Speed Communications (Part II) September 2012, Vol.10 No.3

[22] Xiang Zhou, Lynn E. Nelson, Peter Magill, "PDM-Nyquist-32QAM for 450-Gb/s Per-Channel WDM Transmission on the 50 GHz ITU-T Grid", Journal of Lightwave Technology, Vol. 30,Issue 4,pp. 553-559 (2012)

[23] D.Qian,; M.F.Huang; E.Ip ; Y.K.Huang," High Capacity/Spectral Efficiency 101.7-Tb/s WDM Transmission Using PDM-128QAMOFDM Over 165-km SSMF Within C- and L-Bands", Journal of Lightwave Technology (Volume:30 , Issue: 10 ), Page(s): 1540 - 1548 , 2012

[24] V.A.J.M. Sleiffer, Y. Jung, V. Veljanovski, R.G.H. van Uden, M. Kuschnerov,"73.7 Tb/s (96 x 3 x 256-Gb/s) mode-division-multiplexed DP-16QAM transmission with inline MM-EDFA", Optics Express, vol 20, issue 26 pp. B428-B438, 2012

[25] Xiang ZHOU, "Enabling technologies and challenges for transmission of $400 \mathrm{~Gb} / \mathrm{s}$ signals in $50 \mathrm{GHz}$ channel grid", Front. Optoelectronic (Springer). 2013, 6(1): pp 30-45, 2013

[26] Li, C; Luo, M; Xiao, X; Li, J; He, ZX; Yang, Q; Yang, Z; Yu, SH, "63-Tb/s (368x183.3-Gb/s) C- and L-band all-Raman transmission over 160-km SSMF using PDM-OFDM-16QAM modulation", Chinese Optics Letters 12(4) November 2013

[27] D. G. Foursa, H. G. Batshon, H. Zhang, M. Mazurczyk, J.-X. Cai, " 44.1 Tb/s Transmission over 9,100 km Using Coded Modulation Based on 16QAM Signals at $4.9 \mathrm{bits} / \mathrm{s} / \mathrm{Hz}$ Spectral Efficiency", 39th European Conference and Exhibition on Conference: Optical Communication (ECOC 2013)

[28] Fahad Almasoudi, Khaled Alatawi, Mohammad A. Matin, "1.05 Tb/s Optical-OFDM Using ROF over 3600 km", Optics and Photonics Journal, vol3, pp 318-323, 2013

[29] Jun Sakaguchi, Benjamin J. Puttnam, "305 Tb/s Space Division Multiplexed Transmission Using Homogeneous 19-Core Fiber”, Journal of Lightwave Technology (Volume:31, Issue: 4 ) 2013

[30] L. H. H. Carvalho; E. P. Silva; R. Silva," Spectrally-efficient 17.6-Tb/s DWDM optical transmission system over $678 \mathrm{~km}$ with pre-filtering analysis", Journal of Microwaves, Optoelectronics and Electromagnetic Applications, vol.12 no.2 2013

[31] J. X. Cai ; H. Zhang; H. G. Batshon; M. Mazurczyk, "Transmission over 9,100 km with a capacity of $49.3 \mathrm{~Tb} / \mathrm{s}$ using variable spectral efficiency 16 QAM based coded modulation”, IEEE Optical Fiber Communications Conference and Exhibition (OFC), March 2014, San Francisco, CA

[32] Guifang Li, Neng Bai, Ningbo Zhao, and Cen Xia, Space-division multiplexing: the next frontier in optical communication, Advances in Optics and Photonics, Vol 6 issue 4 pp. 413-487, 2014

[33] Sinan M. Abdul Satar, Mohammed Jalal Abdul-Razzak, "Coherent Optical - OFDM using 64QAM to high data rates $1.60 \mathrm{~Tb} / \mathrm{s}$ over 4500 km”, International Journal of Scientific and Research Publications, Volume 5, Issue 9, September 2015

[34] Talha Rahman, Adriana Lobato, Danish Rafique,"Long-Haul WDM Transmission of $1 \mathrm{~Tb} / \mathrm{s}$ Superchannel" International Conference on Transparent Optical Networks 08/2015; 2015

[35] Xin Xiao; Fan Li; Jianjun Yu," High-speed real-time OFDM transmission based on FPGA ",Optical Metro Networks and Short-Haul Systems VIII, 977304 (13 February 2016)

[36] David S. Millar, Robert Maher, Domaniç Lavery, Toshiaki Koike-Akino," Design of a $1 \mathrm{~Tb} / \mathrm{s}$ Superchannel Coherent Receiver”, Journal of Lightwave Technology Vol. 34, Issue 6, pp. 1453-1463 (2016)

[37] Takayuki Mizuno, Akihide Sano, "Dense Space-Division Multiplexed Transmission Systems Using Multi-Core and Multi-Mode Fiber", Journal of Lightwave Technology, Vol. 34, NO. 2, JANUARY 15, 2016

[38] Shaoliang Zhang, FatihYaman, Yue-Kai Huang, John D. Downie," Capacity-Approaching Transmission over $6375 \mathrm{~km}$ at Spectral Efficiency of 8.3 bit/s/Hz", Optical Fiber Communication Conference 2016, Anaheim, California United States, 20-22 March 2016

[39] Mohammed Abdulmalek Ahmed," Li-Fi: The Future Bright Technology in Wireless Communication", International Journal of Advanced Research in Computer Science and Software Engineering, Volume 6, Issue 3, March 2016

[40] N. Navyatha, T. Prathyusha, V. Roja, and M. Mounika, "Li-Fi (Light fidelity)-LED Based Alternative," International Journal of Scientific \& Engineering Research, vol. 4, 2013

[41] "Harald Haas: Wireless data from every light bulb" 
[42] Mohammed Abdulmalek Ahmed," Li-Fi: The Future Bright Technology in Wireless Communication" International Journal of Advanced Research in Computer Science and Software Engineering, Volume 6, Issue 3, March 2016

[43] http://www.lificonsortium.org/technology.html

[44] http://www.hhi.fraunhofer.de/- Fraunhofer Heinrich Hertz Institute

[45] http://spectrum.iee.org/tlecom/internet/lifi-gets-ready-to-compete-with-wifi

[46] Sebastian Anthony, "Researchers create 3Gbps LiFi network with LED bulbs", International Business Times, May 2013

[47] Li-Fi protocol allows use of the internet at the speed of light-phys.org, July 2014

[48] Li-Fi internet breakthrough: 224Gbps connection broadcast with an LED bulb International Bussiness Times February 2015

[49] A. T. Hussein, M. T. Alresheedi, and J. M. Elmirghani, "20 Gb/s Mobile Indoor Visible Light Communication System Employing Beam Steering and Computer Generated Holograms," Journal of Lightwave Technology, vol. 33, pp. 5242-5260, 2015.

[50] www.lifi-centre.com/lifi-in-the-news-again-the-connected-solar-panel 